\title{
Editorial
}

\section{Spatial-temporal patterns of acute diarrheal disease in south India}

The field of medical geographic information systems (Medical GIS) has become extremely useful in understanding the bigger picture of public health. The discipline holds a substantial capacity to understand not only differences, but also similarities in population health all over the world. The main goal of marrying the disciplines of medical geography, public health and informatics is to understand how countless health issues impact populations, and the trends by which these populations are affected. From the 1990s to today, this practical approach has become a valued and progressive system in analyzing medical and epidemiological phenomena ranging from cholera to cancer. ${ }^{1,2}$ The instruments supporting this field include geographic information systems (GIS), disease surveillance, big data, and analytical approaches like the Geographical Analysis Machine (GAM), Dynamic Continuous Area Space Time Analysis (DYCAST), cellular automata, agent based modeling, spatial statistics and self organizing maps.

In the present work, the spatio-temporal pattern of acute diarrheal disease (ADD) is studied in Chennai during the years 2009-2011. ${ }^{3}$ GIS is used to map ADD data ward-wise. There is very large variation in the cumulative incidence rate of ADD between 155 wards with a minimum of 0 cases $/ 100,000$ per year to a maximum of 1699.7 cases/100,000 per year. The variation in incidence rate is also evident among different age groups as well as among different seasons. Higher incidence is prevailed in northern part of Chennai near Coastal area.

A common index of disease incidence and morbidity is the standardized morbidity ratio (SMR). The SMR is a reliable measure of relative risk for large geographical regions such as countries or states, but may be unreliable for small areas such as municipal wards. ${ }^{3}$ This paper reviews two empirical Bayes methods for producing smoothed estimates of the SMR. A multilevel Poisson gamma and Poisson lognormal model with covariates is developed, and estimating functions are used to estimate model parameters.

The authors are using SMR and the relative risk inadvertently. Since SMR is being used as an estimate of the relative risk under consideration, only SMR should have been used in order to avoid any kind of confusion.

Spatial analysis methods in GIS revealed the spatial patterns and hotspots of ADD in Chennai from the year 2009 to 2011. To implement specific and geographically appropriate public health risk reduction programs, the use of such spatial analysis tools may become an integral component in the epidemiologic description, analysis, and risk assessment of diarrhea.

The positive effects on disease mapping have proven to be tremendous as these instruments continue to have a great impact on the mission to improve worldwide health care. While traditional uses of GIS in public health are static and lacking real time components, implementing a space time animation in these instruments will be monumental as technology and data continue to grow. ${ }^{4}$

\section{References}

1. Glass GE, Aron JL, Ellis JH, Yoon SS. Applications of GIS Technology to Disease Control. The John Hopkins University, School of Hygiene and Public health; 1993 Available from: http://www.popline.org/node/334233 [accessed 17.02.17].

2. Chaikaew N, Tripathi NK, Souris M. Exploring spatial patterns and hotspots of diarrhea in Chiang Mai, Thailand. Int J Health Geogr. 2009;8:36.

3. Kumar VS, Devika S, George S, Jeyaseelan L. Spatial mapping of acute diarrheal disease using GIS and estimation of relative risk using empirical Bayes approach. Clin Epidemiol Global Health. 2016;10.1016/j.cegh.2016.07.004.

4. Meza JL. Empirical Bayes estimation smoothing of relative risks in disease mapping. J Stat Plan Inference. 2003;112:43-62.

Girdhar Gopal Agarwal

Department of Statistics, University of Lucknow, IndiaE-mail address: girdhar1751@gmail.com (G. Agarwal).

Received 21 February 2017

Available online 24 March 2017 